\title{
BISYMMETRIC SPIRAL MAGNETIC FIELDS AND GRAVITATIONAL INSTABILITIES OF GALACTIC DISKS
}

\author{
T. SAWA ${ }^{1}$ and M. FU JIMOTO ${ }^{2}$ \\ ${ }^{1}$ Department of Earth Sciences, Aichi University of Education, \\ Kariya, Aichi 448, Japan \\ 2 Department of Physics, Nagoya University, \\ Nagoya 464-01, Japan
}

\begin{abstract}
Linearized equations of motion are solved for the self-gravitating gaseous disk in interaction with the Lorentz force due to the bisymmetric spiral (BSS) magnetic fields. Since the pattern velocity of the BSS fields is very close to that of the spiral density wave, a nearly-resonant interaction occurs between these two waves to enhance spiral condensation of gas. The BSS fields seem to compensate the spiral density waves for their secular dispersion.
\end{abstract}

\section{The BSS Magnetic Fields and Galactic Dynamo}

Evolution of large-scale magnetic fields $B$ in spiral galaxies is described by the induction equation,

$$
\frac{\partial \boldsymbol{B}}{\partial t}=\operatorname{rot}(\boldsymbol{V} \times \boldsymbol{B})+\eta \nabla^{2} \boldsymbol{B}+\operatorname{rot}(\alpha \boldsymbol{B})
$$

with $\operatorname{div} B=0$, where $\boldsymbol{V}$ denotes the galactic rotation velocity, $\eta$ the turbulent diffusion constant, and $\alpha$ the dynamo strength. Equation (1) has been solved for the BSS magnetic fields by Sawa and Fujimoto (1986) and Baryshnikova et al. (1987). The former authors obtained the $z$-distribution of $\boldsymbol{B}$ by assuming its radial distribution, and the latter the radial distribution by using the local dynamo

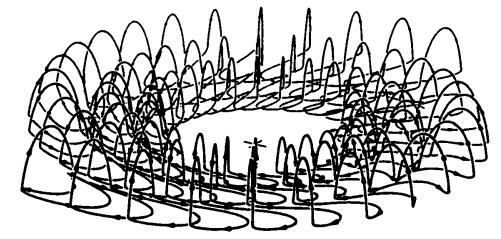

Fig. 1. The bird's eye view of the magnetic lines of force. The upper half of the field lines are shown. solution for the $z$-distribution of $B$.

We search for an asymptotic solution of $B$ in the BSS form of $B=b(r) \exp [i\{-\omega t+$ $k z+\phi+(1 / \epsilon) \ln r\}$, where $b(r)$ is a slow function of $r$ and $\epsilon$ denotes the pitch angle of the BSS field. It is found that the BSS field pattern can be maintained when $\alpha$ and $h$, the half thickness of the gaseous disk, are distributed as in Table 1 (Fujimoto and Sawa 1987). Figure 1 shows the three-dimensional configuration thus obtained of the BSS fields (Sawa and Fujimoto 1987).

\begin{tabular}{ccccccc}
\multicolumn{5}{l}{ Table $1 . \alpha$ and $h$ for a steady BSS field in $r=4 \sim 12 \mathrm{kpc}\left[\operatorname{Re}(\omega)=13 \mathrm{~km} \mathrm{~s}^{-1} \mathrm{kpc}^{-1}\right]$} \\
\hline$r(\mathrm{kpc})$ & 4.0 & 5.5 & 7.0 & 8.5 & 10.0 & 11.5 \\
$\alpha\left(\mathrm{km} \mathrm{s}^{-1}\right)$ & -5.6 & -4.2 & -3.1 & -2.4 & -1.7 & -1.1 \\
$h(\mathrm{kpc})$ & 0.23 & 0.29 & 0.38 & 0.47 & 0.60 & 0.80 \\
\hline
\end{tabular}

2. Magnetogravitational Interaction between the BSS Magnetic Fields and the Spiral Density Waves

The Lorentz acceleration $\boldsymbol{L}$ due to the BSS fields is extremely small, compared with the background gravitational acceleration. It is, however, able to perturb the galactic rotation of gas when, as Fujimoto (1987) and Lesch et al. (1988) have shown, the angular velocity of the BSS field is similar to that of the spiral density wave. On the basis of this consideration, Fujimoto and Sawa (1989) have solved the following linearized equations of motion for a 
disk of self-gravitating gas permeated with the BSS magnetic fields,

$$
\begin{aligned}
& \frac{\partial \delta \boldsymbol{V}}{\partial t}+(\boldsymbol{V} \cdot \nabla) \delta \boldsymbol{V}+(\delta \boldsymbol{V} \cdot \nabla) \boldsymbol{V}=-\frac{c_{s}^{2}}{\rho} \nabla \delta \rho-\nabla \delta \Psi+\boldsymbol{L}, \\
& \text { and } \quad \frac{\partial \delta \rho}{\partial t}+\operatorname{div}(\rho \delta \boldsymbol{V}+\boldsymbol{V} \delta \rho)=0,
\end{aligned}
$$

with $\boldsymbol{L}=\left(L_{r}, L_{\phi}\right)=\operatorname{rot} B \times B / 4 \pi \rho$, and $\nabla^{2} \delta \Psi=$ $4 \pi G \delta \rho$. Writing the Lorentz accelerations and the perturbed quantities in the form of $\hat{q} \exp \left[2 i\left\{-\Omega_{p} t+\right.\right.$ $\phi+(1 / \epsilon) \ln r\}]$, the amplitude of the perturbed gas density is given by

$$
\frac{\hat{\rho}}{\rho}=\frac{1}{D}\left(-\frac{2 i}{\kappa^{2} \epsilon r} \hat{L}_{r}+\frac{4 \Omega}{\nu} \frac{1}{\kappa^{3} \epsilon r} \hat{L}_{\phi}\right),
$$

with $D=1-\nu^{2}+4 c_{s}^{2} /(\kappa \epsilon r)^{2}-(4 \pi G)(2 h \rho) / \kappa^{2} \epsilon r, \Omega=$ $V / r$, and $\nu=2\left(\Omega_{p}-\Omega\right) / \kappa$, where $c_{s}$ is the velocity dispersion of turbulence, $\kappa$ the epicyclic frequency, and other symbols have their usual meanings.

When $\boldsymbol{B}=0$ or $\boldsymbol{L}=0$, we have $D=0$. The condition $D=0$ is exactly a dispersion relation for the spiral density wave (Lin and Shu 1964), in which, however, $\Omega_{p}$ must be replaced with $\Omega_{s}$, the angular velocity of the density wave. Since $\Omega_{p} \simeq \Omega_{s}$, we have $|D| \simeq 0$ in equation (4) and thus a nearly resonant interaction occurs to produce stronger condensation of gas in a double spiral way. For the BSS fields in figure 2, it is on the spiral loci joining the local maxima (or minima) of $\left|B_{\phi}\right|$ when the disk with $B=0$ is gravitationally unstable (or stable) against the spiral perturbation, i.e. $D<0$ (or $D>0$ ).

\section{Concluding Remarks}

We have searched for theoretically the BSS magnetic fields in spiral galaxies and found that they are maintained to rotate rigidly without changing their global configuration. Since the BSS field pattern rotates with approximately the same angular velocity as the spiral density waves, these two waves interact strongly and enhance the spiral condensation of gas. If the galactic disk is composed entirely of self-gravitating gas, the secular dispersion of the density waves (Toomre 1969) could be supplied with this magnetogravitational condensation of gas.

\section{References}

Baryshnikova, Y., Ruzmaikin, A., Sokoloff IV, D.D., and Shukurov, A. (1987) Astron. Astrophys. 177, 27-41.

Fujimoto, M. (1987) in Interstellar Magnetic Fields, R. Beck and R. Gräve (eds), Springer Verlag, Berlin, 23-29.

Fujimoto, M. and Sawa, T. (1987) Publ. Astron. Soc. Japan 39, 375-392.

Fujimoto, M. and Sawa, T. (1989) Geophys. Astrophys. Fluid Dynamics, in press.

Lesch, H., Sawa, T., Krause, M., Beck, R., Fujimoto, M., and Biermann, P.L. (1988) Astron. Astrophys. 192, 19-23.

Lin, C.C. and Shu, F.H. (1964) Astrophys. J. 140, 646-655.

Sawa, T. and Fujimoto, M. (1986) Publ. Astron. Soc. Japan 38, 133-150.

Sawa, T. and Fujimoto, M. (1987) in Magnetic Fields and Extragalactic Objects, E. Asséo and D. Grésillon (eds), Editions de Physique, 165-169.

Toomre, A. (1969) Astrophys. J. 158, 899-913. 